\title{
Author Index for Volume 22
}

\begin{tabular}{|c|c|c|c|}
\hline Abuhlail, J. & 47 & Guo, W.B. & 437 \\
\hline Albeverio, S. & 541 & Guo, X.J. & 11 \\
\hline Alhevaz, A. & 271 & Guo, Y.Q. & 445 \\
\hline Ali, U. & 561 & Habibi, M. & 271 \\
\hline Amin, I. & 655 & Haider, A. & 561 \\
\hline Andreeva, D.P. & 437 & Han, G.Q. & 639 \\
\hline Anwar, I. & 707 & Hassanzadeh, S.H. & 671 \\
\hline Azam, F. & 561 & Ibrahim, Y. & 655 \\
\hline Azam, S. & 621 & Jahangiri, M. & 131 \\
\hline Bahlekeh, A. & 469 & Javaid, I. & 561 \\
\hline Bonacini, P. & 97 & Jiang, Q.F. & 517 \\
\hline Borna, K. & 671 & Jiang, W. & 233 \\
\hline Button, J.O. & 333 & Karpuz, E.G. & 35 \\
\hline Chen, H.Y. & 413 & Kashif, A. & 707 \\
\hline Chen, H.-Y. & 479 & Kim, S. & 395 \\
\hline Chen, J.L. & 169 & Lee, D.-I. & 1 \\
\hline Cuenca Mira, J.A. & 603 & $\mathrm{Li}, \mathrm{F}$. & 677 \\
\hline de Giovanni, F. & 181 & Li, H.S. & 459 \\
\hline Delgado, J.M.S. & 83 & Li, J.B. & 581 \\
\hline Dinh, L.V. & 421 & Li, Y.L. & 169 \\
\hline Erfanian, A. & 449 & Liang, L. & 259 \\
\hline Farrokhi D.G., M. & 449 & Liao, H.-Y. & 687 \\
\hline Foth, P. & 395 & Liu, H.G. & 555 \\
\hline Gao, M. & 367 & Liu, K.-S. & 163 \\
\hline Gao, X.C. & 309 & Liu, W.D. & 309 \\
\hline Gao, Y.Y. & 169 & Liu, Y.F. & 211 \\
\hline Gao, Z.Z. & 495 & Liu, Z. & 383 \\
\hline Grašič, M. & 109 & Manaviyat, R. & 607 \\
\hline Gu, W.P. & 73 & Marino, L. & 97 \\
\hline
\end{tabular}


Martín, A.J.C.

Moussavi, A.

Nguyen, H.N.

Nikouei, M.

Obul, A.

Olteanu, A.

Omirov, B.A.

Ouyang, B.Y.

Pan, J.M.

Pei, J.

Qureshi, A.A.

Raposo, A.P.

Raza, Z.

Ren, C.C.

Rinaldo, G.

Rozikov, U.A.

Salimi, M.

Shang, Y.L.

Shirmohammadi, N.

Shum, K.P.

Skiba, A.N.

Song, G.A.

Sun, L.P.

Tahamtan, Sh.

Tan, D.Z.

Tavasoli, E.

Teh, J.-H.

Tong-Viet, H.P.

Tribak, R.

Trombetti, M.

Tvalavadze, M.

Viet, D.Q.

Vorob'ev, N.T.

Wakefield, T.P.
Wang, S.

517,711

271,607

293

621

495

23

541

349

383

445

567

321

707

11

223

541

215

361

131

11, 445

437

251

309

131

677

215

687

293

119

181

281

421

211

293
Wang, Y.L.

555

309

73

251

251

Xin, B.

367, 405, 581

Yáñez, M.F.

189

Yang, C.H.

259

Yao, H.L.

639

Yassemi, S.

215

211

Yousif, M.

655

$\mathrm{Yu}, \mathrm{H}$.Y.

73

$\mathrm{Yu}, \mathrm{X} . \mathrm{F}$.

383

Yue, X.Q.

367, 405

Yunus, G.

495

349

147

Zheng, R.D.

711
Yi, X.L. 